\title{
The Research into Structure-dependent Gas-Solid Two-phase Flow within Vertical Screw Conveyor
}

\author{
Sun Xiaoxia ${ }^{1}$, Meng Wenjun ${ }^{2}$, Leung, R. C. K. ${ }^{3}$ and Yuan yuan ${ }^{4}$ \\ Mechanical Engineering College, Taiyuan University of Science and Technology, \\ Taiyuan, Shanxi 030024, P.R. China \\ ${ }^{1} 860115787 @ q q . c o m,{ }^{2}$ tyustmwj@126.com, ${ }^{3}$ randolph.leung@polyu.edu.hk, \\ 4yuanyuan525622@126.com
}

\begin{abstract}
This paper proposes a type of multi-scale structure of gas-solid DEM model by analyzing the gas-solid two-phase flow model in a vertical screw conveyer. The model considers the non-uniform flow structure feature in the vertical screw conveyor, which decomposes the flow structure into two homogeneous phases: dilute phase and dense phase. The movement rule of gas is described with a two-phase coupled Navier-Stokes equation, of which the particle movement rule is described with Newton's second law. The drag force of the gas-solid two-phase flow is based on the EMMS model. This paper examines the influence of particle size, screw speed, and filling rate on vertical speed and concentration distribution of the particle, which is combined with FLUENT software and EDEM software for simulation analysis of this model. The result shows that as maller particle size corresponds to higher screw speed; as maller filling rate corresponds to a more conducive formation of gas-solid two-phase flow in vertical screw conveyor and vertical conveyance of particles.
\end{abstract}

Keywords: vertical screw conveyor, gas-solid two-phase flow, DEM model, EMMS model

\section{Introduction}

In China, the design and manufacturing technology of screw ship unloaders is in a poor state because of two main reasons. The first reason isthe study of basic theory of conveying mechanisms. The second reason involves problems in theoretical and structural designs, material selection, and manufacturing technology of high-efficiency screw conveyor with theoretical basis. The design theory and method are especially important.

The traditional screw conveying mechanism indicates that the horizontal screw conveyor can only convey granular media at extremely low rotation speeds (as if dead nuts move rectilinearly along the rotating threaded rod), but the vertical screw conveyor must convey the granular media upward at an extremely high rotation speed. Both types of conveyance are achieved based on friction.

Air flow field can be generated in the space of closed homocentric cylinders that rotate when facing each other [1]. The high-speed screw rotating in the cylinder can also affect the air to generate a flow field in the space of closed homocentric cylinders. As the granule gaps in granular media are filled with air, when the screw blade rotates at a relatively low speed, it has a minimal impact on the granular media and air around it. Such an effect cannot generate an effective flow field; when the rotation speed of the screw blade is relatively high, the air is impacted by the screw blade while generating flow fields in the closed space [2-3]. If design and control are reasonable, a stable screw vortex occurs and generates suction, thereby resulting in intake of the granular media into the screw conveyor [4-7]. Fluid motion with both rotation and radial motion is called a 
vortex, which plays a role in enhancing the axial velocity of conveyed granular media and generates extremely high-density gas-solid two-phase flow to significantly improve conveying efficiency.

As early as the1980s, research on vertical screw conveyors had been conducted; key points were proposed to design new types of vertical screw conveyors and the irmain technical parameters, which laid a foundation for further research [8]. Yan Liu was among the first to apply mechanics of granular media to vertical screw ship unloaders by adopting the so-called material group method that analyzes coefficient of lateral compressive stress and distribution of lateral compressive stress, which is applied to the wall of material tank and screw shaft by granular media. However, when this method was used to deduce the curvilinear equation of free surface, the an elasticity of granular media was neglected in this method; an elasticity occurs in the actual situation [9]. Using the method of Yan Liu, a team led by Yongzhi Li applied further improvements by analyzing the stress state of granular media in the vertical screw conveyor and confirming the stress function of granular media. Their team further obtained curvilinear equation and theoretical criterion of free surface relating to filling rate and determined a computational formula of positive pressure under different boundary conditions [10]. Compared with the material group method, this method considers the an elasticity of granular media when the curvilinear equation of free surface of granular media is confirmed. Furthermore, research on the pressure distribution of granular media under different boundary conditions is implemented in this method by adopting the method of mechanics of granular media; this method is closer to the actual conveying circumstance. However, interaction within the granular media is neglected in this method in which actual description of working condition of the vertical screw conveyor cannot be implemented. At present, many scholars are investigating screw conveying mechanisms by adopting the single mass point method, in which the screw blade is simplified as a bevel that spreads following the helical line and the granular media as a single mass point on the bevel; the force and movement of this mass point are investigated [11-13]. The internal applied force of the granular media and distribution of its lateral pressure are not considered in this method. When the filling rate and conveying capacity are relatively high and large, respectively, the calculated result has a relatively large difference from the actual circumstance. The air flow field that is generated when screw rotation is performed, together with its effect on conveyance of granular media material, has not been considered in the literature.

Theoretical and experimental research into screw conveying mechanisms had already been initiated even before the development of screw ship unloaders. Scholars from Germany, including Vierling and Sinha, were the first to propose single mass-point theory of the screw conveying mechanism. In this theory, the granular media on helicoid is simplified as a single mass point to analyze its motion and applied force. The theory also introduces the concept of critical rotation speed to bring up the analytical method of solving the ascending speed of granular media, thereby further confirming the conveying capacity and power of the vertical screw conveyor. Its disadvantage is that the diversity composed of granules of granular media is neglected, as is then pressure distribution characteristic of the granular media on the pipe wall and screw surface of the vertical screw conveyor. However, this method does not comply with the actual circumstance of a screw ship unloader with high filling rate. Therefore, based on the theory of hydromechanics, the granular media was abstracted as an ideal fluid by the Swedish scholar Nilsson and the Finnish scholar Rademacher. Thus, the analytical solution of free surface and pressure distribution of the granular media was obtained. However, the disadvantage of this solution is that the essential difference between the granular media and the ideal fluid is neglected, which inevitably results in an error between the theoretically analyzed and actual conclusions. Thus, application of this theory is limited. In terms of the boundedness of the aforementioned theory, with granule group regarded as the subject, the German scholars Gabier and Bttcher proposed that stacking state of the 
granular media on helicoid changes as the screw rotation speed changes. Thus, the theoretical result is much closer to reality but still does not comply with the actual circumstance of the screw ship unloader with high filling rate [14].

\section{Structure-Dependent Gas-Solid Two-Phase Model}

\subsection{Motion Density of Material Within Vertical Screw Conveyor}

The process of conveying the material within a vertical screw conveyor was analyzed based on medium mechanics and fluid mechanics; the motion density distribution of the material is obtained. The motion density is 0.5 to 0.8 times the packing density near the screw axis because of the effect of centrifugal force. The motion density is 1.008 to 1.018 times the packing density in the middle of the screw axis and wall section. A sharp increase in motion density occurs near the pipe wall; the material compression on the pipe wall and the absolute velocity and the axial velocity drop rapidly. Thus, motion density is uneven in the vertical screw conveyor material characterized by radial heterogeneity of nuclear structure, which has a rare center and a thick wall.

\subsection{Structure-Dependent Gas-Solid Two-Phase DEM Model (SGSDM Model)}

The vertical screw conveyor system is a typical complex system; its main characteristic is a heterogeneous multi-scale structure. The traditional average method considers the vertical screw conveyor system as a uniform system that averages all the parameters. However, the average method cannot correctly reflect the basic features of inhomogeneous flow structure of the vertical screw conveyor system; thus, the real behavior inside the system cannot be described. Such systems cannot achieve quantization. The multi-scale method of considering the structural characteristics is effective in quantitative calculation for this type of system.

Flow structure of the vertical screw conveyor can be decomposed into two homogeneous phases, namely dilute and dense. The dilute phase is composed of single uniform particles suspended in the gas, and the dense phase is composed of particles that collide with one another. The interaction between these two phases can result in exchanges in quality. The dilute and dense phases are composed of gas and particles, so interaction (drag force)occurs between the gas and particles in each phase. In addition, interaction occurs between the two phase; quality exchange occurs between dilute phase gas and dense phase gas, and quality exchange between dilute phase particles and dense phase particles. Furthermore, quality exchange occurs between dilute phase gas and dense phase particles (drag force). Thus, a structure-dependent gas-solid two-phase DEM model is built for particles in a vertical screw conveyor. The gas-solid DEM model indicates that when addressing the gas-phase field with Eulerian method at the same time, discrete particle field with Lagrangian method is dealt with by directly tracking particles in the particulate flow.

\subsubsection{Control Equation for Gas Phase}

The mass conservation equationfor dense phase gas is

$$
\frac{\partial\left(f \mathcal{E}_{g c} \rho_{g}\right)}{\partial t}+\nabla \cdot\left(f \varepsilon_{g c} \rho_{g} u_{g c}\right)=M_{g}
$$

The mass conservation equationfor dilute phase gas is

$$
\frac{\partial\left[(1-f) \varepsilon_{g f} \rho_{g}\right]}{\partial t}+\nabla \cdot\left[(1-f) \varepsilon_{g f} \rho_{g} u_{g f}\right]=-M_{g}
$$

The momentum conservation equationfor dense phase gas is 


$$
\frac{\partial\left(f \varepsilon_{g c} \rho_{g} u_{g c}\right)}{\partial t}+\nabla \cdot\left(f \varepsilon_{g c} \rho_{g} u_{g c} u_{g c}\right)=-f \varepsilon_{g c} \nabla_{p}+\nabla \cdot\left(f \varepsilon_{g c} \tau_{g}\right)+f \varepsilon_{g c} \rho_{g} g-f F_{d c}
$$

The momentum conservation equationfor dilute phase gas is

$$
\begin{gathered}
\frac{\partial\left[(1-f) \varepsilon_{g f} \rho_{g} u_{g f}\right)}{\partial t}+\nabla \cdot\left[(1-f) \varepsilon_{g f} \rho_{g} u_{g f} u_{g f}\right] \\
=-(1-f) \varepsilon_{g f} \nabla_{p}+\nabla \cdot\left[(1-f) \varepsilon_{g f} \tau_{g}\right]+(1-f) \varepsilon_{g f} \rho_{g} g-(1-f) F_{d f}-F_{d i} \\
\because \varepsilon_{g}=f \varepsilon_{g c}+(1-f) \varepsilon_{g f} \\
\varepsilon_{g} u_{g}=f \varepsilon_{g c} u_{g c}+(1-f) \varepsilon_{g f} u_{g f} \\
S_{p}=\beta\left(u_{g}-u_{s}\right)=f F_{d c}+F_{d i}+(1-f) F_{d f} .
\end{gathered}
$$

$\therefore(1)+(2)$ to the following mass conservation equation for gas phase:

$$
\frac{\partial\left(\varepsilon_{g} \rho_{g}\right)}{\partial t}+\nabla \cdot\left(f \varepsilon_{g} \rho_{g} u_{g}\right)=0
$$

(3) $+(4)$ to the following momentum conservation equation for gas phase:

$$
\frac{\partial\left(f \varepsilon_{g} \rho_{g} u_{g}\right)}{\partial t}+\nabla \cdot\left(\varepsilon_{g} \rho_{g} u_{g} u_{g}\right)=-\varepsilon_{g} \nabla_{p}+\nabla \cdot\left(\varepsilon_{g} \tau_{g}\right)+\varepsilon_{g} \rho_{g} g-S_{p}
$$

\subsubsection{Control Equation For Particles Phase}

The particle flow is treated as discrete phasedescribed by DEM, whether in the process of particlecollisions of the dense phaseorin the process of suspended particles ofthedilute phase.

Particles aremainly influenced by gravity, centrifugal force, forcebetween particles and particles, forcebetween particles and wall surface, forcebetween particles and screw vane, drag force, pressure gradient force, buoyancy, added mass force, Basset force, Saffman force, and Magnus force. The effect of buoyancy can be ignored because the magnitude of the ratio of buoyancy and gravity is 10:3. The particledoes not undergothe effect of added mass force and Basset force because the particle motion ofthescrew conveyor is constant.The effects of Saffman force and Magnus force on gas particles is lowcompared with the effects of gravity, drag force, and collision force.

When thevertical screw conveyormovesparticles, the particles aremainly influenced bygravity, centrifugal force, drag force, pressure gradient force, force between particles and particles, force between particles and wall surface, and force between particles and the screw vane. The control equation for the particles phase is as follows:

$$
\begin{gathered}
m \frac{d u_{s}}{d t}=m g+\frac{m u_{s}^{2}}{r}+F_{d}+F_{b}+F_{p-p}+F_{p-w}+F_{p-s} \\
I \frac{d w}{d t}=\sum_{j=1}^{k_{i}} T_{i j}
\end{gathered}
$$

wherem, $I, u_{s}$, and $\omega$ represent quality, moment of inertia, average speed, and

rotational speed of particles in the dense phase, respectively; mg is gravity, and $\frac{m u_{s}^{2}}{r}$ is centrifugal force.

The expression of air drag force is as follows:

$$
F_{d}=\frac{V_{p} \beta}{1-\varepsilon}\left(u_{g}-u_{s}\right)
$$

where $V_{p}$ is the particle volume, $\varepsilon_{\text {is }}$ the void fraction, ${ }^{u_{g}}$ is the gas velocity, $u_{s}$ is the particle velocity, and ${ }^{\beta}$ is the momentum transfer coefficient between the gas-solid two phase, which is acquired from the EMMS analysis process, as shown in formula (15).

The pressure gradient force is as follows: 


$$
F_{b}=-V_{p} \nabla_{p}
$$

where $V_{p}$ is the particle volume and $\nabla_{p}$ is the pressure gradient on the particle.

$F_{p-p}$ is the force between particles and particles, $F_{p-w}$ is the force between particles and the wall surface, and $F_{p-s}$ is the force between particles and the screw vane. The soft ball model is used to describe the force between particles, which allows deformation of the particle in the process of collision and multi-body collision. This modelis suitable for high concentrations of the impact of particles inavertical screw conveyor.

The soft ball model is as follows:

$$
F_{p-p}=\sum_{j=1}^{k_{i}}\left(F_{n, i j}+F_{t, i j}\right)
$$

where $F_{n, i j}, F_{t, i j}$ are respectivelythe normal force and the tangential force thatparticle $i$ suffered from particle $j$. $F_{n, i j}=\left(-k_{n} \delta_{n}^{1.5}-\eta_{n j} G \cdot n\right) n, \quad F_{t, i j}=-k_{t} \delta_{t}-\eta_{t j} G_{c t}$.

The soft ball model is also used to describe the force between particles and the wall surface as well asthe force between particles and the screw vane. When considering the effect of particles and the wall throughthe soft ball model, the wall can be assumed as a particle with zerovelocity, with infinite diameter, and withoutdisplacement. When considering the effect of particles and the screw vaneviathe soft ball model, the screw vane can be assumed as a particle whose velocity isthescrew speed, with infinite diameter, andwithout displacement.

Under the action of the aforementioned force and moment, thespeed, rotating speed, and position changes of the particle areas follows:

$$
\begin{gathered}
V_{i}=V_{i, 0}+\frac{d V_{i}}{d t} \Delta t \\
\omega_{i}=\omega_{i, 0}+\frac{d \omega_{i}}{d t} \Delta t \\
x y z_{i}=x y z_{i, 0}+V_{i} \Delta t
\end{gathered}
$$

\subsubsection{Drag Force Model in SGSDM Model}

In the particle movement process, the particles suffer drag force from gas, and gas affects the particle flow because of the existence of particles; thus,tight coupling is observed between the two roles.The density of particle flow is uneven in themovementprocess in the screw conveyor. Most existing drag force models arebased on the average method to calculate the momentum exchange coefficient between the two phases, such as the Wen and Yu/Ergun models; these models cannot accurately reflect the gas-solid interaction mechanism of the heterogeneous system. Thus, perfecting and developingthe drag force model between the gas and solid phasesis necessary based on multi-scale structure. This modelis applied to numerical simulation of the dense gas-solid two-phase flow in the screw conveyor, thereby obtainingthe distribution of gas and particles and other useful parameters. These parameters includespeed of gas and particles, motion curve, lifting height, average mass flow rate, power, and energy consumption during collisionbetween particles and particles, wall surface, annd screw vane.

The drag force model based on EMMS considersthe law of energy conservation of each scale. These scales consider the influence of the pressure insidethegrid and the change in kinetic energy of gas in allsimulated calculationunits, therebysuccessfully solving the non-uniform flow characteristics of the gas-particle two-phase mechanism. Thus, analysis ofnon-uniform flow state of gas-solid two-phase flow in the vertical screw conveyor was improved.

The calculation process of the multi-scale structure gas-solid two-phase DEM model combined with EMMS is as follows: average flow variables ${ }_{g}, u_{s},{ }_{\varepsilon_{g}}$ of the local grid 
are provided by fluid computation of CFD and EDEM, respectively, which areinput conditions of EMMS analysis. Heterogeneous two-phase state variables $\beta$ in the computational grid areobtained by EMMS viaflow decomposition and energy analysis, which areused to close the multi-scale structure of the gas-solid two-phase DEM model.

The expression of the state variable $\beta$ based on theEMMS drag model is as follows:

$$
\beta=\left\{\begin{array}{cc}
\frac{3}{4} \frac{\left(1-\varepsilon_{\mathrm{g}}\right) \varepsilon_{\mathrm{g}}}{\mathrm{d}_{\mathrm{p}}} \rho_{\mathrm{g}}|\mathrm{u}-\mathrm{v}| \mathrm{C}_{\mathrm{d} 0} \cdot \omega\left(\varepsilon_{\mathrm{g}}\right) & \varepsilon_{\mathrm{g}}>0.74 \\
150 \frac{\left(1-\varepsilon_{\mathrm{g}}\right)^{2} \mu_{\mathrm{g}}}{\varepsilon_{\mathrm{g}} \mathrm{d}_{\mathrm{p}}{ }^{2}}+1.75 \frac{\left(1-\varepsilon_{\mathrm{g}}\right) \rho_{\mathrm{g}}|\mathrm{u}-\mathrm{v}|}{\mathrm{d}_{\mathrm{p}}} \varepsilon_{\mathrm{g}} \ll 0.74
\end{array},\right.
$$

where theexpression of structure modification factor $\omega\left(\varepsilon_{g}\right)$ is as follows:

$$
\omega\left(\varepsilon_{\mathrm{g}}\right)=\left\{\begin{array}{cl}
-0.5760+\frac{0.0214}{4\left(\varepsilon_{\mathrm{g}}-0.7463\right)^{2}+0.0044} & 0.74<\varepsilon_{\mathrm{g}} \leq 0.82 \\
-0.0101+\frac{0.0038}{4\left(\varepsilon_{\mathrm{g}}-0.7789\right)^{2}+0.0040} & 0.82<\varepsilon_{\mathrm{g}} \leq 0.97 \\
-31.8295+32.8295 \varepsilon_{\mathrm{g}} \varepsilon_{\mathrm{g}}>0.97
\end{array}\right.
$$

\section{Simulation Process}

\subsection{Simulation Parameter Settings}

To simulate the actual particle transmission, the conveyor adopts the feeding head method, and the initial parameters are as follows: radius of spiral blade $R l=0.1 \mathrm{~m}$, diameter of screw axis is $0.032 \mathrm{~m}$, thickness of screw blade $\Delta h=0.002 \mathrm{~m}$, pitch $S=0.1 \mathrm{~m}$, diameter of screw tube wall $R 2=0.112 \mathrm{~m}$, and height of screw conveyor $h=1.2 \mathrm{~m}$. Modelling followed a shrink ratio of 1:4.

The model built via Solid Works software is imported into EDEM software, as shown in Figure 1.

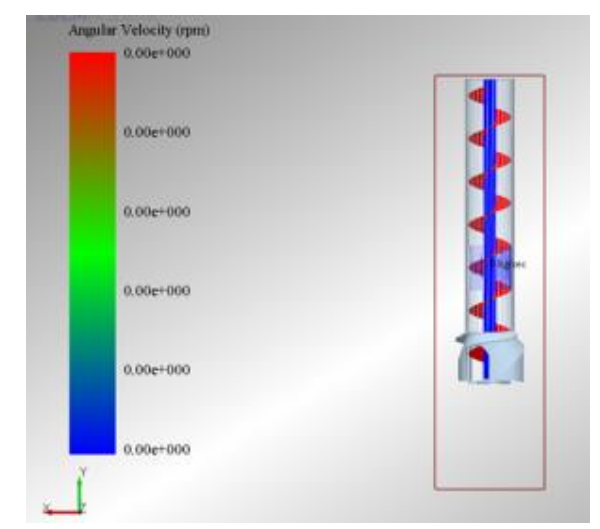

Figure 1. Calculation Model for Vertical Screw Conveyor

\subsection{Calculation Procedure}

The simulation program of the gas-solid DEM model based on the multi-scale structure(SGSDM model) constitutes FLUENT calculation of the gas-phase field and EDEM calculation of particle dynamics. During simulation, the solutions of the particle-phase field and gas-phase field are alternated. The specific steps are as follows:

(1)The gas-phase flow field and particle flow field are initialized. 
(2) A numerical solution is used to control equations (5) and (6) of gas phase within the given time step using the SIMPLEC method, and to obtain the velocity field of the gas phase.

(3)The particle movement is solved as follows:

The air drag force and pressure gradient force are solved according to equations(9)and(10), respectively. The occurrence of collision whether between particles and particles, the wall, and the screw blade is determined. If collision occurred, the impact force is calculated based on equation (11). Then, the force and moment that affect the particles are calculated based on equation(7). Then, the acceleration of particle collision is determined based on Newton's second law. Finally, the position and speed of all particles after a time step are calculated based on equations(12), (13), and (14) to determine the motion curve of particles.

(4) The void fraction in the gas-phase flow field grid is updated as the average speed of particles in the grid is computed, and the reaction source term of $S_{p}$ of the particle to flow field is calculated.

(5) The gas-phase control equation is amended, and then the new gas-phase flow field is concluded.

(6) Steps (2)-(5) are repeated until the end of the step length.

\section{Simulation Results}

\subsection{Influence of Particle Size on Particle Vertical Speed}

The gas-solid two-phase DEM simulation is based on multi-scale structures for different particle sizes. Comparison charts of vertical speed for different sizes of particle and gas are shown in Figures 2 to5.

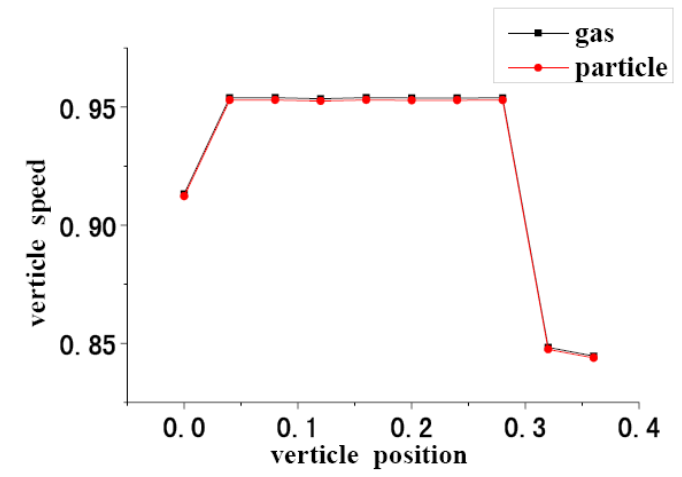

Figure 2. Vertical Speed of Particle and Gas with Particle Size of $10 \mathrm{Mm}$ 


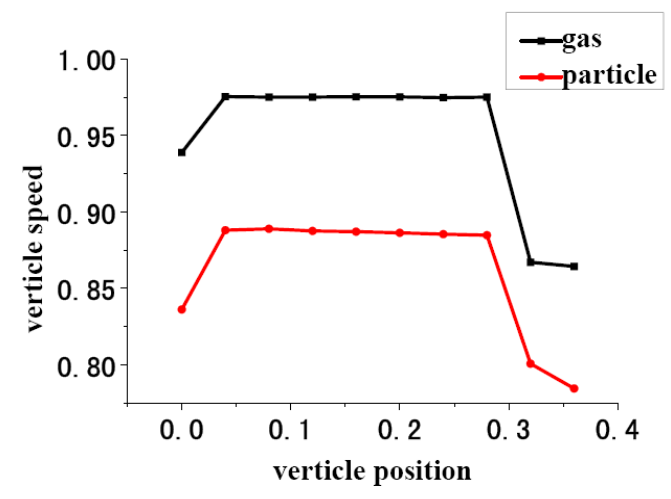

Figure 3. Vertical Speed of Particle and Gas with Particle Size of $100 \mathrm{Mm}$

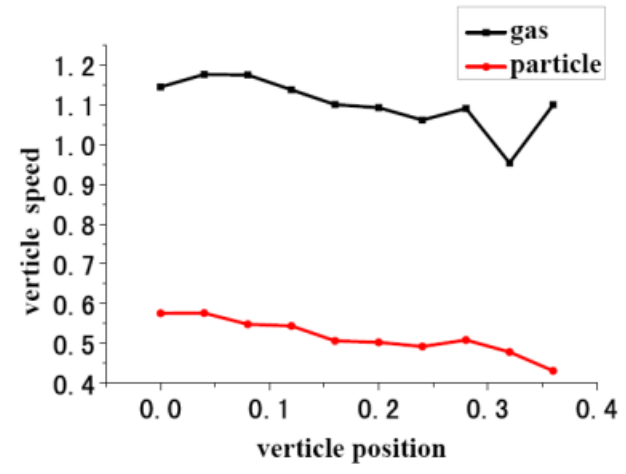

Figure 4. Vertical Speed of Particle and Gas with Particle Size of $500 \mathrm{Mm}$

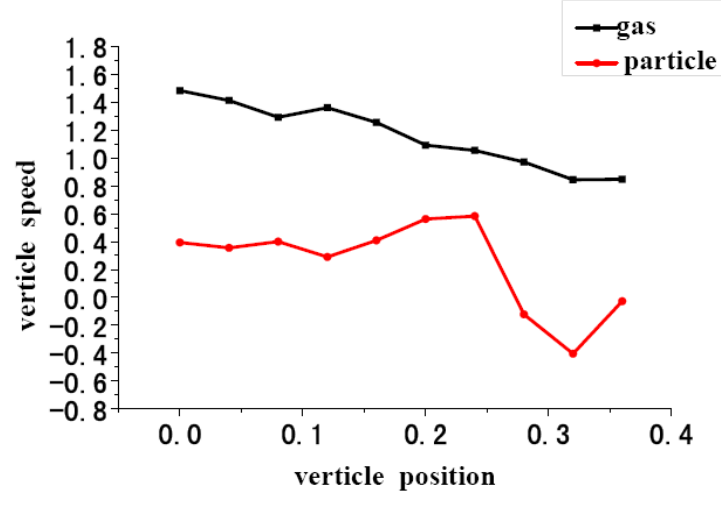

\section{Figure 5. Vertical Speed of Particle and Gas with Particle Size of 1000 Mm}

As shown in Figures 2 to 5, with the increase of particle size, the airflow speed that was consistent with particle speed gradually decreased. When the particle size is $10 \mu \mathrm{m}$, the airflow speed is consistent with the particle speed. When the particle size is $100 \mu \mathrm{m}$, the particle speed and airflow are in the same direction, but the airflow speed is significantly greater than the particle speed. When the particle size is $500 \mu \mathrm{m}$, the particle speed and the airflow indicate a small difference in the same direction, and the relative speed is greater. When the particle size is $1000 \mu \mathrm{m}$, the particles peed and airflow show a significant difference in the same direction, and the relative speed is greater. Such a result indicates that the greater the particle size is, the smaller is the influence of air flow on the particle. In the delivery process, the friction between the particle and wall surface and 
screw vane is the main consideration. The gas can push the particle transport when the particle size is at the micron scale. In this scenario, the main consideration is improving deferent speed by flow field distribution.

\subsection{Influence of Screw Speed on Particle Vertical Speed}

The multi-scale structure of the gas-solid two-phase DEM simulation is obtained based on different screw speeds, as well as the contrast figure of vertical speed of particle and gas with different screw speed, as shown in Figures 6 to8.

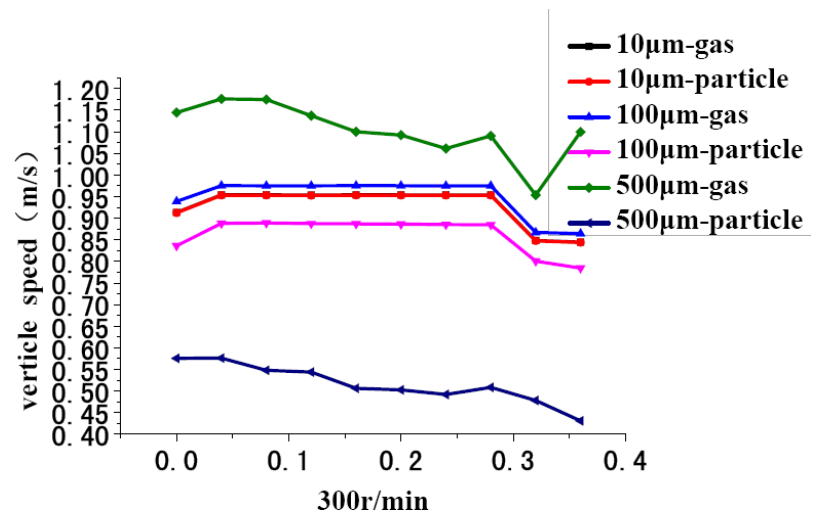

Figure 6. Vertical Speed of Particle and Gas with Different Particle Sizes, With Screw Speed of 300 R/Min

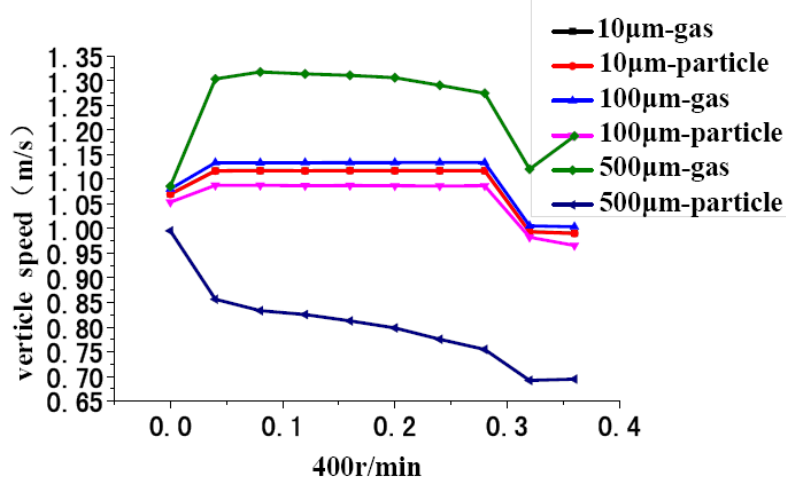

Figure 7. Vertical Speed of Particle and Gas with Different Particle Sizes, With Screw Speed of $\mathbf{4 0 0}$ R/Min 


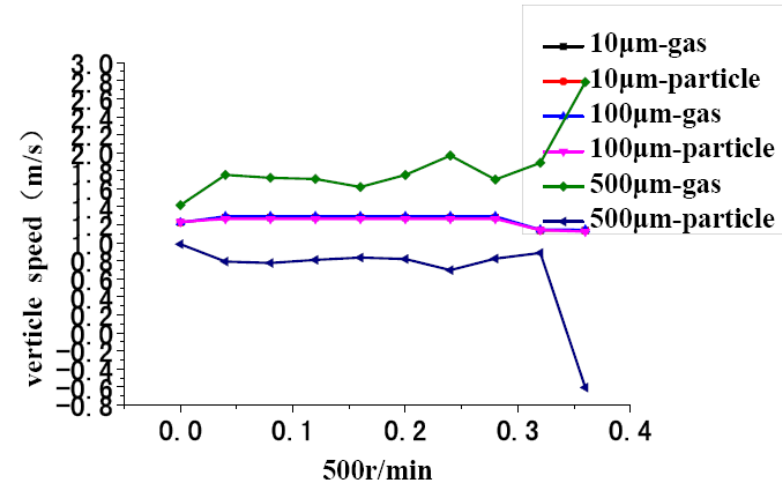

\section{Figure 8. Vertical Speed of Particle and Gas with Different Particle Sizes,} With Screw Speed of 500 R/Min

The vertical speeds of particle and corresponding gas with different particle sizes are shown in Figures 6 to 8; screw speeds are 300, 400, and 500 r/min, respectively. The graph indicates that the speed of gas and particle is consistent with the screw speed of $300 \mathrm{r} / \mathrm{min}$ and the particle size of 10 microns, whereas the speed of gas and material is consistent, but has larger relative speed when the particle size is 100 microns. When the screw speed is $400 \mathrm{r} / \mathrm{min}$, the speed curve of particle and gas remains the same with the particle size of 10 microns, whereas the relative velocity of particle and gas decreases with the particle size of 100 microns. When the screw speed is $500 \mathrm{r} / \mathrm{min}$, the speed curve of particle and gas remains the same as the particle size of $100 \mu \mathrm{m}$, and coincides with the speed curve which has a particle size of $10 \mu \mathrm{m}$. Under the a fore mentioned three types of screw speed, the particle is obviously different from the direction of the gas, and the speed difference is also significant when the particle size is 500 microns. However, the relative velocity of the particle and gas with the screw speed of $500 \mathrm{r} / \mathrm{min}$ is less than the screw speeds of $300 \mathrm{r} / \mathrm{min}$ and $400 \mathrm{r} / \mathrm{min}$.

Following these specifications, the screw speed of screw aid in the conveyance of particles. The faster the screw speed, the smaller the relative velocity between the gas and particle, thereby resulting in motion resistance of particles. The screw speed can be increased to improve the efficiency of transportation when transporting larger particles.

\subsection{Influence of Filling Rate of Particle on Particle Vertical Speed}

The vertical speeds of particles with different filling rates are shown in Figure 9. With the increase of filling rate, the vertical speed of particles decreases instead. The reason is that the greater the filling rate of particles, the greater the number of particles; the more complicated collision of particles, the more interaction occurs between material and gas. These scenarios can reduce the material conveying speed instead. 


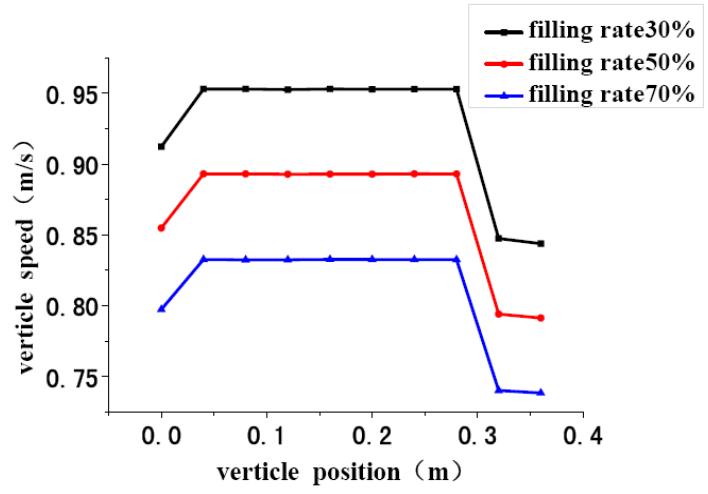

Figure 9. Vertical Speed of Particle with Different Filling Rates

\subsection{Influence of Particle Size on Particle Concentration Distribution}

As shown in Figure 10, the larger the particle size, the smaller the particles under the influence of gas, and the particle aggregation phenomenon becomes more obvious. This condition is particularly evident when the particle size is 500 microns; in this scenario, the main consideration is the friction effect between particles and conveyor pipe wall in the process of transmission.

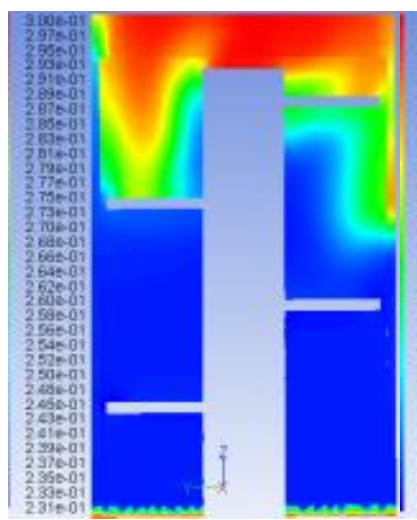

(a) $30 \%$

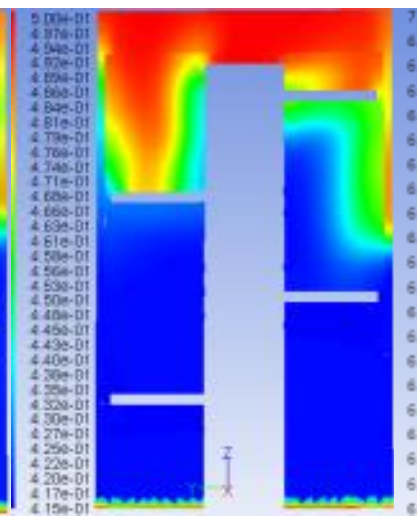

(b) $50 \%$

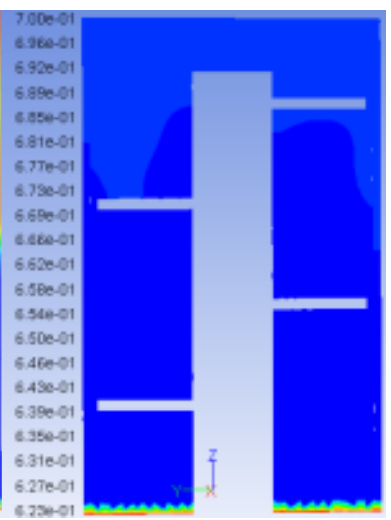

(c) $70 \%$

Figure 10. Concentration Distribution with Three Types of Filling Rate

\subsection{Influence of Screw Speed on Particle Concentration Distribution}

Figure 11 shows the concentration distribution of the particle under three types of screw speed, i.e., 300, 400, and $500 \mathrm{r} / \mathrm{min}$, respectively, with particle size of $10 \mu \mathrm{m}$. The greater the screw speed, the more severe the particle motion in the conveyor; the interaction of particle with gas is also fuller. 


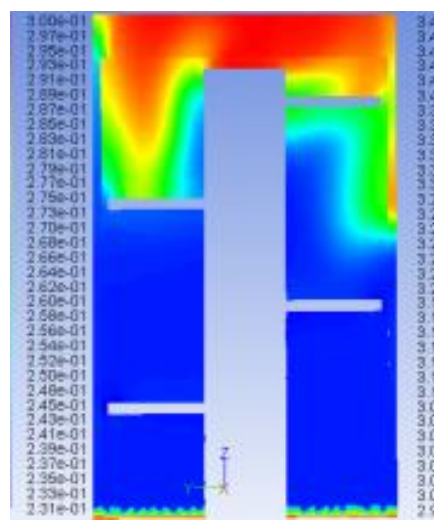

(a) $300 \mathrm{r} / \mathrm{min}$

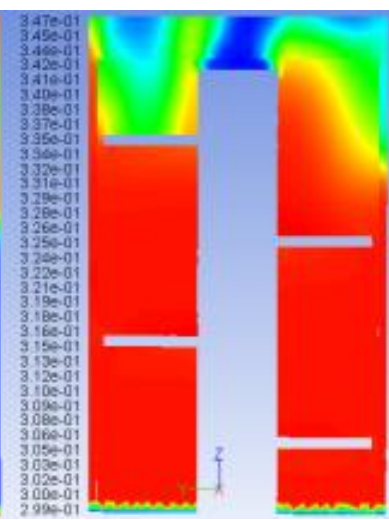

(b) $400 \mathrm{r} / \mathrm{min}$

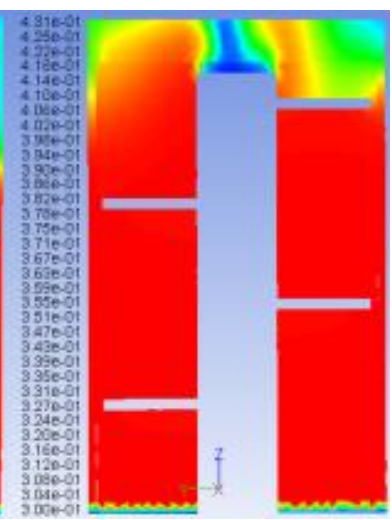

(c) $500 \mathrm{r} / \mathrm{min}$

Figure 11. Concentration Distribution with Three Types of Screw Speed

\subsection{Influence of Filling Rate of Particle on Particle Concentration Distribution}

Figure 12shows the concentration distribution of the particle under three types of screw speed of 300, 400, and $500 \mathrm{r} / \mathrm{min}$, respectively, whose particle size is $10 \mu \mathrm{m}$. When the filling rate is $30 \%$, anobvious difference is observed in the high concentration area and low concentration area of the particle, thereby indicating that the conveyor is not full of particles. When the filling rate is $50 \%$, minimal change is observed in the concentration distribution with $30 \%$. When the filling rate is $70 \%$, minimal difference is observed in the high-concentration and low-concentration areas of the particle, whose concentration distribution is balanced. Thus, the particle accounts for a higher proportion in the conveyor.

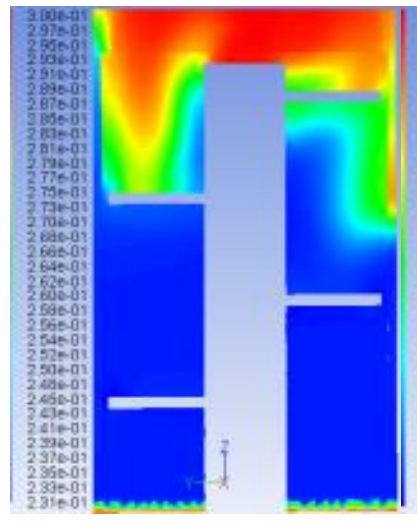

(a) $30 \%$

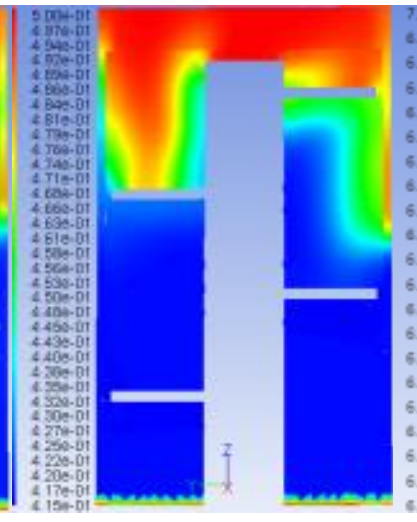

(b) $50 \%$

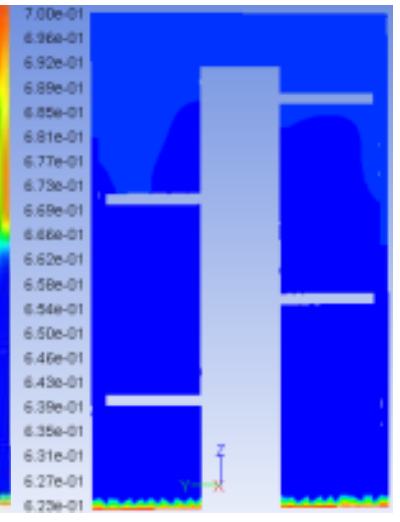

(c) $70 \%$

Figure 12. Concentration Distribution with Three Types of Filling Rate

\section{Conclusion}

(1) This paper proposes a type of multi-scale structure of gas-solid DEM model (SGSDM model)that is suitable for a vertical screw conveyor. This structure can correctly reflect the basic features of uniform flow structure within the vertical screw conveyor system and describe the real behavior within the system.

(2) The influence factors on the vertical speed of the particle are obtained via simulation analysis for the model combined with FLUENT and EDEM software. The effect of particle size and screw speed is the largest: the greater the particle size, the 
smaller the ascending velocity of the particle; the higher the screw speed, the larger the ascending velocity of the particle. Second largest is the filling rate of the particle. The higher the filling rate, the smaller the particle speed, which means that a higher filling rate is not better. Comprehensive consideration of various conditions is needed. Finally, for large particle size to achieve high speed need, screw speed must be improved.

(3) The influence factors on the concentration distribution of the particle are obtained via simulation analysis for the model combined with FLUENT software and EDEM software. The smaller the particle size, the more uniform the particle in the gas distribution, thereby resulting in a scenario that is more conducive to transport. The faster the screw speed, the higher the degree of particle mixing with gas. The higher the filling rate of particles, the less obvious the concentration distribution of particles.

\section{Acknowledgment}

This studywas supported by the National Natural Science Fund Project under Grant No. 51575370, the Shanxi International Cooperation Project under Grant No. 2015081008, theShanxi Jincheng Science and Technology Plan Project under Grant No. 201501004-7, and the Shanxi Coal-based Key Scientific Research Project under Grant Nos. MJ-201402 and MJ-201409.

\section{References}

[1] Y.-T. Wang, Y.-F. Qiao and G. Yang, "Methods of gas-solid two-phase flow velocity measurement", Control and Decision Conference (CCDC), (2011); China.

[2] X.-Y. Yin and D.-J. Sun, “The Stability of Vortex Flow”, National Defense Industry Press, (2003), pp. 485-490.

[3] S. Benzarti, H. Mhiri and H. Bournot, "Drag models for Simulation Gas-Solid Flow in the Bubbling Fluidized Bed of FCC Particles", World Academy of Science, Engineering and Technology, (2012), pp. 1138-1143.

[4] M. Sadegh and M. Shirazi, "Mal-distribution of Gas-solid flow Through Identical Parallel Paths", The Faculty of Graduate Studies, The University of British Columbia, (2009), pp. 312-320.

[5] D.-P. Shi, Z.-H. Luo and A.-Y. Guo, "Numerical Simulation of the Gas-Solid Flow in Fluidized-Bed Polymerization Reactors", Ind. Eng. Chem. Res, (2010), pp. 4070-4079.

[6] X.-J. Liu and L. Shi, "Current Situation of Research into Dense Gas-solid Two-phase Flow with Euler-Lagrange Method", Academic Journal of Computer Mechanics, vol. 24, (2007), pp. 166-172.

[7] Y.-L. Ma, "Numerical Simultation and Study of High-density Gas-solid Two-phase Flow", Paper for Docterate of Zhejiang University, (2001); China.

[8] W.-K. Chen, "A Kind of New-Type Vertical Screw Conveyor", First Issue Number 87, Academic Journal of Hunan College of Sciences and Arts, vol. 24, (2014), pp. 90-92.

[9] Q.-Z. Jiang and Y. Liu, "Mathematical Model and Optimal Design of Conveying Mechanism of Screw Ship Unloader", Hoisting and Conveying Machinery, vol. 2, (1989), pp. 2-9.

[10] Y.-Z. Li, H.-L. Bi and Q.-Z. Jiang, "Research Into Vertivcal Conveying Process of Screw Ship Unloader", Academic Journal of Wuhan Water Transportation Engineering College, vol. 6, (1992), pp. 131-136.

[11] S.-M. Huang, "Conveying Mechanism of Screw Conveyor and Confirmation of Its Main Parameters", Guangdong Papermaking, vol. 3, (1998), pp. 27-31.

[12] S.-W. Zhou, "The Application of Screw Conveyor in Cigarette Making Machine", Academic Journal of Hunan College of Sciences and Arts, vol. 24, (2012), pp. 90-92.

[13] X.-F. Liang, "Development and Application of New-type Vertical Screw Conveyor", Sulfur and Phosphorus Design and Powder Engineering, vol. 3, (2003), pp. 45-47.

[14] J.-G. Cheng and W.-J. Meng, "Research into Mobility of Granular Media inside the Vertical Screw Conveyor", Mechanical Engineering and Automation, vol. 6, (2012), pp. 1-3. 


\section{Authors}

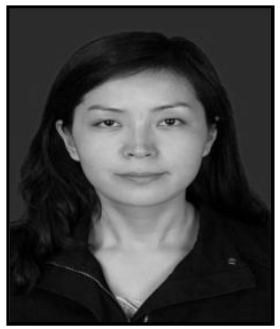

Sun Xiaoxia, received her B.Eng. degree in computer and its application in 2002, M. Eng. degree in computer integrated manufacturing in 2005, both from North University of China(NUC), China. Currently, she is a Ph.D. candidate at Taiyuan University of Science and Technology(TYUST), China. Her research interests include the design of the continuous conveying machinery. 